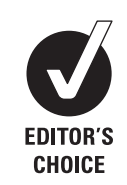

\section{See Editorial, pg 1175}

'Department of Rheumatology, Sint Maartenskliniek, Nijmegen, The Netherlands 2Department of Rheumatology, Radboud University Nijmegen Medical Centre, Nijmegen,

The Netherlands

\section{Correspondence to} Gijs F Snijders, Sint Maartenskliniek, Department of Rheumatology, PO Box 9011, 6500 GM, Nijmegen, The Netherlands; gijssnijders@gmail.com

Accepted 21 March 2011 Published Online First 8 May 2011

\title{
The effects of doxycycline on reducing symptoms in knee osteoarthritis: results from a triple-blinded randomised controlled trial
}

\author{
Gijs F Snijders, ${ }^{1}$ Cornelia HM van den Ende, ${ }^{1}$ Piet LCM van Riel, ${ }^{2}$ \\ Frank HJ van den Hoogen, ${ }^{1}$ Alfons A den Broeder ${ }^{1}$; on behalf of NOAC study group
}

\begin{abstract}
Objectives Evidence suggests that doxycycline might have disease-modifying properties in osteoarthritis. However, the clinically relevant question as to whether doxycycline also modifies symptoms in knee osteoarthritis is unanswered. The objective of this study was to investigate the effectiveness of doxycycline on pain and daily functioning in symptomatic knee osteoarthritis. Methods A 24-week, randomised, triple-blind, placebo controlled trial on the symptomatic effectiveness of doxycycline twice a day $100 \mathrm{mg}$ in knee osteoarthritis patients according to the clinical and radiological American College of Rheumatology classification criteria. The primary endpoint was the difference in the proportion of participants in both study groups achieving a clinical response defined by the OMERACT-OARSI set of responder criteria. Secondary endpoints included pain, stiffness, daily functioning, patient global assessment, quality of life, osteoarthritis-related medication and side effects.
\end{abstract}

Results 232 patients were randomly assigned. At study end, $31 \%$ of participants met the primary endpoint in both groups. Except for more adverse events in the doxycycline group, no differences were also found on the secondary endpoints.

Conclusions Doxycycline is not effective in reducing symptoms in knee osteoarthritis patients over a 24-week study period, but is associated with an increased risk of adverse events. Although a possible structure-modifying effect of doxycycline was previously suggested, this is not accompanied by symptom relief in the short and medium term.

Dutch Trial Register no NTR1111.

The search for a disease-modifying osteoarthritis drug (DMOAD) for osteoarthritis targeting both symptoms and structure has been intensified in recent years. Several studies investigating candidate $\mathrm{DMOAD}$ in clinical trials have been published, including dietary supplements, diacerein, strontium ranalate, bisphosphonates, biological agents, autologous conditioned serum, calcitonin and doxycycline. ${ }^{1-18}$

However, up to now, no agent is known to be an irrefutable confirmed DMOAD as results of most studies remain largely ambiguous or difficult to interpret. For diacerein, risedronate, calcitonin and autologous conditioned serum the efficacy on symptoms have been demonstrated in randomised controlled trials, but these results have thus far not been confirmed in more than one study, except for diacerein. Effects on structural change have been shown for risedronate, diacerein and doxycycline, 51213 although these results have not been replicated successfully thus far. ${ }^{19}$ Whether doxycycline also modifies symptoms of osteoarthritis has not yet been established.

Doxycycline is a tetracycline class antibiotic agent. Besides being an antimicrobial agent, it is a metalloproteinase inhibitor and inhibits the collagenase that cleaves collagen type IX that is present in articular cartilage. ${ }^{20-22}$ Doxycycline has been studied in human osteoarthritis in one clinical trial, in which doxycycline was found to retard the progression of radiographic knee osteoarthritis. ${ }^{13}$ The original intent of this study was to assess the efficacy of doxycycline to retard progression and prevent the occurrence of radiographic knee osteoarthritis in overweight female patients with unilateral radiographic knee osteoarthritis. However, although the contralateral knee was radiographically normal in the conventional anterior-posterior (AP) view, in most cases there was evidence of osteoarthritis in the lateral, semiflexed AP and/or patellofemoral view. Therefore, de facto the effect of doxycycline on the progression of less extensive osteoarthritis in that joint was assessed. Although no effect on less extensive knee osteoarthritis was seen, radiographic progression was substantially slower in the knee with established osteoarthritis. No effect on pain was found, presumably due to low pain scores at enrolment. However, post-hoc analyses demonstrated a lower incidences of increased pain $>20 \%$ increase in pain score) in the index knee but not in the non-affected knee. Also, side effects were mild, and drop-out due to the possible side effects of doxycycline was rare $(\sim 8 \%)$ during the 30 -month trial period.

Although a proof of principle of DMOAD properties of doxycycline has thus been demonstrated, the clinically relevant question as to whether doxycycline also reduces pain and improves daily functioning in symptomatic knee osteoarthritis has not been answered.

To explore the effects of doxycycline on reducing symptoms in knee osteoarthritis, a 24-week tripleblinded, randomised, placebo controlled trial was conducted to evaluate the effect of doxycycline on pain and daily functioning in well-established knee osteoarthritis.

\section{METHODS}

\section{Study design}

This triple-blinded, randomised, placebo controlled trial was performed as a mono-centre study in the Sint Maartenskliniek, Nijmegen, The Netherlands. 
The local Medical Research Ethics Committee, region Arnhem/ Nijmegen, The Netherlands, and the national Central Committee on Research involving Human Subjects (CCMO) approved the study. Moreover, the study was registered at EUDRACT and in the Dutch Trial Register (www.trialregister.nl; trial number: NTR1111). All participants gave their informed consent.

\section{Participants}

Patients were eligible for inclusion if they met the following criteria: fulfilment of the clinical and radiological American College of Rheumatology classification criteria for knee osteoarthritis ${ }^{23}$ in the index knee (defined as the knee causing most complaints during the screening visit), Kellgren and Lawrence (K\&L) score 2 or $3,{ }^{24}$ knee injury and osteoarthritis outcome score-derived Western Ontario McMaster Universities (WOMAC) score pain subscale (see details below) of greater than 20/100 and the ability to read and communicate well in Dutch. Exclusion criteria were: inflammatory rheumatic diseases or deposition diseases possibly leading to inflammatory arthritis or secondary osteoarthritis, extensive orthopaedic abnormalities (eg, major malalignment $\left(>5^{\circ}\right)$ ), co-morbidity exceeding complaints of limitations of the knee, cognitive or sensomotor problems interfering with the use of questionnaires or intake of study medication, planned other major interventions within 24 weeks (including lower limb surgery and intensive multidisciplinary approaches), hip prosthesis in situ on the side of the symptomatic knee, contraindications for doxycycline use, such as allergy for tetracyclines and previous possible adequate treatment with doxycycline (>100 mg/day for $>6$ weeks for osteoarthritis), recent intra-articular hyaluronic acid/corticosteroid application or arthroscopy ( $<3$ months) or open surgical procedures ( $<1$ year) in the index knee.

Participants were allowed to use analgesics during the study period, but they were asked to stop these agents during the last $48 \mathrm{~h}$ and/or four times the drugs half-life before the study visits at the outpatient clinic (baseline, weeks 12 and 24). Opioids other than tramadol (up to $150 \mathrm{mg} /$ day) were not allowed.

Participants were recruited from the rheumatology and orthopaedics outpatient clinics and from advertisements in local newspapers.

\section{Setting}

All visits and collection of data took place at the rheumatology outpatient clinic of the Sint Maartenskliniek, Nijmegen, The Netherlands, a hospital specialising in rheumatology, orthopaedics and rehabilitation.

\section{Randomisation and intervention}

Eligible patients were randomly assigned (allocation ratio 1:1) to receive either $100 \mathrm{mg}$ of oral doxycycline monohydrate or placebo twice a day (one in the morning and one in the evening) for 24 weeks. The allocation was blinded for patient and study physician (GFS) using placebo medication capsules, blue and white, with the same appearance as verum. Participants who violated the study protocol were encouraged to adhere to study visits to limit loss to follow-up.

An independent pharmacist used a computer-generated, blinded randomisation list to assign patients randomly to doxycycline or placebo. Allocation data were stored at the hospital pharmacy in sealed envelopes that could be opened in the case of medical need. To increase balance in possible confounders, allocation was stratified for intensity of pain (moderate vs severe, ie, $<60$ vs $\geq 60$ on the WOMAC pain subscale, respectively) at the screening visit using stratified block randomisation. Assignment of patients to the right stratum of the random assignment list was performed by the study physician (GFS) who was blinded to therapy.

\section{Assessments}

Visits were planned at screening (week -2), baseline, weeks 12 and 24 at the outpatient clinic and in weeks 6 and 18 by telephone. The following data were collected:

- Baseline characteristics: demographics, duration of complaints, previous osteoarthritis-related treatments, concomitant medication.

- Radiographs (at screening): bilateral (posterior-anterior fixed flexion and lateral) knee radiographs were performed and graded using the K\&L grading scale by the study physician (GFS).

- Questionnaires (at screening, baseline, week 12 and week 24): to estimate knee osteoarthritis-related symptoms patients were asked to fill out the Dutch version of the knee injury and osteoarthritis outcome score (Likert scale version) questionnaire. ${ }^{25}$ This questionnaire includes the WOMAC score ${ }^{26}$ in its complete and original format (with permission, http:// www.koos.nu). WOMAC pain, stiffness and function subscales were presented as normalised scores (0-100, where 0 equals no symptoms). To assess quality of life, the short form 36 (SF-36) ${ }^{27}$ questionnaire was completed by all participants. The SF-36 consists of eight subscales with a score range of $0-100$, where 100 represents the best possible health situation. The physical component summary (PCS) and mental component summary (MCS) scores were calculated as weighted means of the four physical and four mental subscale scores, respectively (higher scores indicate better health situation).

- Patient global assessment: visual analogue scale-patient global assessment (VAS-PGA), 0-100, where 0 equals no symptoms (at screening, baseline, week 12 and week 24).

- (Changes in) osteoarthritis related medication use (during all visits).

- Adverse events: during all follow-up visits patients were asked if they experienced any possible adverse events, which were graded for severity. Any adverse event resulting in death, hospitalisation, prolongation of hospitalisation, or development of a life-threatening or debilitating condition was categorised as a serious adverse event (SAE). Routine laboratory testing (baseline and week 24) included liver and renal function in addition to blood cell counts and vitamin B12 (the latter only at week 24).

- Therapy adherence: pill counts (weeks 12 and 24). Adherence to therapy was defined as the use of $80 \%$ or more of the total number of study capsules.

\section{Outcome}

The primary endpoint was the difference in the proportion of participants in both study groups achieving a clinical response at week 24 defined by the OMERACT-OARSI set of responder criteria, ${ }^{28}$ based on the WOMAC pain and function subscale scores and VAS-PGA. The OMERACT-OARSI criteria are defined as: (1) improvement in WOMAC pain $(0-100)$ or WOMAC function $(0-100)$ of $50 \%$ or greater with an absolute change of 20 or greater; or (2) improvement of $20 \%$ or greater with an absolute change of 10 or greater in at least two of the following measures: WOMAC pain, WOMAC function and VAS-PGA. Incomplete questionnaires at baseline 
were replaced by data from the screening visit ( $t=-2$ weeks) if possible. In addition, incomplete questionnaires at study end (week 24) were replaced by data from the 12 -week visit in participants who were still taking study medication at the end of the study (week 24). Participants who ceased study medication prematurely due to adverse events and were lost to follow-up were classified as non-responders. This analysis was performed in all subjects who underwent randomisation but also preplanned in subgroups with and without severe pain (intention-to-treat analysis).

Secondary endpoints included differences between the two study groups at weeks 12 and 24 in (change in) the WOMAC subscales, VAS-PGA, MCS and PCS subscales of the SF-36, nature and frequency of adverse events and the (change in) use of osteoarthritis-related medication at study end.

\section{Sample size calculation}

A difference of $20 \%$ response between the placebo and doxycycline group (number needed to treat 5) was considered to be clinically relevant. ${ }^{28}$ An OMERACT-OARSI response at 24 weeks in the placebo group of $40 \%$ was anticipated. ${ }^{28}$ Together with a requested power of $80 \%$, a two-sided significance level of 0.05 , an allocation ratio of $1: 1$ and an expected drop-out at week 24 of $15 \%, 115$ patients were required per treatment arm.

\section{Statistical analysis}

Statistical analyses were performed using STATA/IC 10.1 for Windows. Descriptive statistics were provided by using mean (SD), median (25th-75th percentiles) or frequencies/percentages depending on distribution of the data. All analyses were performed blinded for treatment allocation.

Analysis of the primary endpoint was carried out using the intention-to-treat principle and was assessed using Fisher's exact test. Change scores were calculated by subtracting the baseline scores from the scores at weeks 12 and 24, respectively. Differences between both study groups in change scores of WOMAC subscales, VAS-PGA, PCS and MCS were analysed using the unpaired t test. Changes in the use of osteoarthritisrelated medication during the trial were categorised in 'more', 'less' and 'unchanged usage' and analysed using the MannWhitney $U$ test. The proportions of adverse events in both treatment groups were compared using Fisher's exact test.

Additional analyses were performed regarding the proportion OMERACT-OARSI responders in participants who completed the trial per protocol.

The imputation of missing values of data of patients lost to follow-up was considered not appropriate as it was anticipated that loss to follow-up selectively occurred in the doxycycline group due to adverse effects (missing not at random). Individuals with incomplete data on the primary outcome were classified as non-responders. To calculate change scores (secondary endpoints) only non-missing data were utilised.

\section{RESULTS}

Between April 2008 and April 2010232 of the 305 patients screened were enrolled and randomly assigned in this study (figure 1). Subjects in the doxycycline group reported slightly higher scores on the WOMAC subscales and concomitant use of analgesics compared with the placebo group. With respect to other possible confounding variables such as demographic variables, body mass index and K\&L grade both study groups were comparable at baseline. Thirty-seven patients had severe pain (table 1).

Among the 232 subjects who were randomly assigned, 204 $(88 \%)$ completed the trial per protocol. Significantly more participants in the doxycycline group compared with the placebo group discontinued the study medication prematurely (21 vs seven, $\mathrm{p}=0.001)$. Twelve participants were lost to follow-up (seven doxycycline vs five placebo group). Adherence to treatment was satisfactory (ie, $\geq 80 \%$ study capsules taken) in $80 \%$ of subjects who were still taking study medication at the final study visit (week 24) and was comparable between both study groups: $80 \%$ versus $80 \%$ in the doxycycline and placebo groups, respectively $(p=1.0)$.

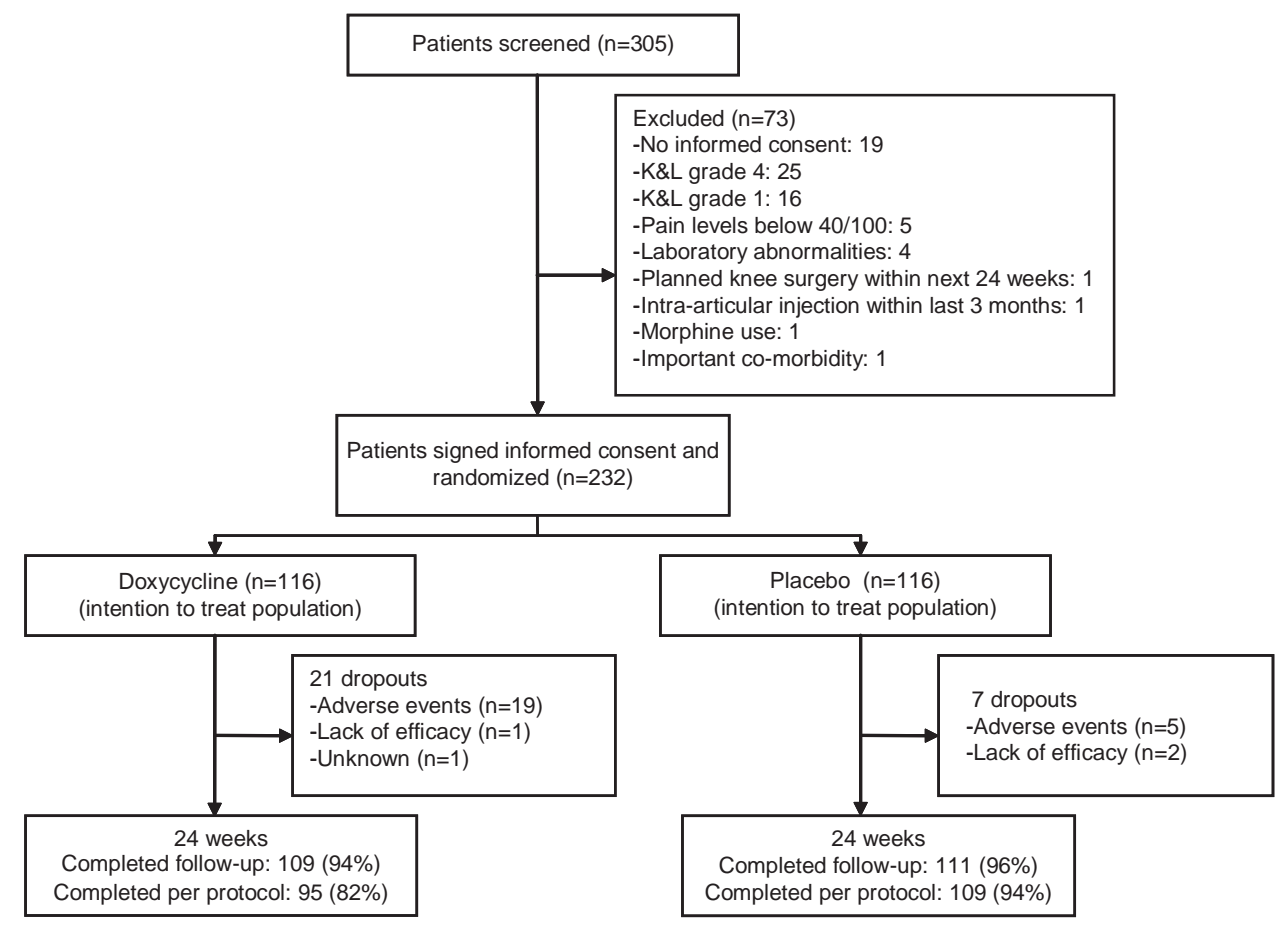

Figure 1. Study flow diagram 


\section{Primary outcome measure}

A total of 72 out of 232 (31\%) participants met the OMERACTOARSI criteria for treatment response at the final study visit (week 24). In the doxycycline and placebo group, 31/116 (27\%) and $41 / 116(35 \%)$ met responder criteria at study end, respectively $(p=0.2)$. In participants with severe pain at week 24 , nine

Table 1 Baseline characteristics of patients

\begin{tabular}{|c|c|c|c|}
\hline Variable & $\begin{array}{l}\text { Total study } \\
\text { population } \\
(n=232)\end{array}$ & $\begin{array}{l}\text { Doxycycline } \\
(n=116)\end{array}$ & $\begin{array}{l}\text { Placebo } \\
(n=116)\end{array}$ \\
\hline Women, n (\%) & $154(66)$ & $79(68)$ & $75(65)$ \\
\hline Age (years), mean (SD) & $59(9)$ & $59(9)$ & $59(10)$ \\
\hline BMI, mean (SD) & $30(5)$ & $30(6)$ & $30(5)$ \\
\hline $\begin{array}{l}\text { Duration of knee complaints (years), } \\
\text { median (p25-75) }\end{array}$ & $6(3-15)$ & $6(3-14)$ & $6(3-15)$ \\
\hline K\&L score $=2$, number $(\%)$ & $151(65)$ & $76(66)$ & $75(65)$ \\
\hline K\&L score $=3$, number (\%) & $81(35)$ & $40(34)$ & $41(35)$ \\
\hline \multicolumn{4}{|l|}{ WOMAC score, mean (SD) } \\
\hline Pain & $49(18)$ & $52(19)$ & $46(17)$ \\
\hline Function & $49(18)$ & $52(18)$ & $47(17)$ \\
\hline Stiffness & $56(21)$ & $58(22)$ & $54(19)$ \\
\hline Severe pain, $\mathrm{n}(\%)^{*}$ & $37(16)$ & $19(16)$ & $18(16)$ \\
\hline VAS-PGA, mean (SD) & $52(25)$ & $52(25)$ & $53(26)$ \\
\hline \multicolumn{4}{|l|}{ Pain medication use, number $(\%)^{\dagger}$} \\
\hline Paracetamol & $81(35)$ & $50(43)$ & $31(27)$ \\
\hline NSAID & $95(41)$ & $51(44)$ & $44(38)$ \\
\hline Tramadol & $12(5)$ & $4(3)$ & $8(7)$ \\
\hline None & $85(37)$ & 34 (29) & $51(44)$ \\
\hline
\end{tabular}

${ }^{*}$ WOMAC pain subscale $\geq 60 / 100$.

${ }^{\dagger}$ Including on-demand use.

BMI, body mass index; K\&L score, Kellgren-Lawrence grading scale; NSAID, nonsteroidal anti-inflammatory drug; VAS-PGA, visual analogue scale-patient global assessment; WOMAC Western Ontario McMaster Universities score. responders were identified (two of 19 in the doxycycline group vs seven of 18 in the placebo group). Again, no significant difference in the proportion of responders was identified between the doxycycline and placebo groups $(p=0.06)$.

\section{Secondary outcome measures}

In the total study population, a significant improvement of symptoms was observed at week 24. Scores on the WOMAC subscales pain, stiffness and function, and VAS-PGA decreased from 49 to $41(n=218), 56$ to $50(n=215), 49$ to $42(n=210)$ and 52 to $44(\mathrm{n}=200)$, respectively (all $\mathrm{p}<0.001)$. Regarding quality of life, PCS improved from 36 to 38 ( $p<0.0001)$, whereas MCS showed no change (53 vs $54, \mathrm{n}=213, \mathrm{p}=0.5$ ). However, no differences between the two study groups were found in any measures (table 2).

\section{Concomitant use of osteoarthritis-related medication}

Of the 204 participants who were still taking study medication at the study end, 153 had unchanged, 23 had increased and 28 had decreased their osteoarthritis-related medication use at week 24. For the doxycycline and placebo groups these numbers were $68,12,15$ and $85,11,13$, respectively, and showed no significant difference between these groups $(\mathrm{p}=0.9)$.

\section{Safety}

During the study, $56 \%$ of the participants reported at least one adverse event. Of the 28 subjects who prematurely ceased study medication, 24 did so because of adverse events. Compared with the placebo group, significantly more participants who were

Table 2 Secondary outcomes: improvements and differences between study groups

\begin{tabular}{|c|c|c|c|c|c|}
\hline & & Doxycycline & & Placebo & Difference \\
\hline Outcome measure & $\mathrm{n}$ & Mean $(95 \% \mathrm{Cl})$ & $\mathrm{n}$ & Mean $(95 \% \mathrm{CI})$ & $(95 \% \mathrm{Cl})$ \\
\hline \multicolumn{6}{|l|}{ WOMAC pain } \\
\hline Baseline (SD) & 116 & $52(19)$ & 115 & $46(17)$ & \\
\hline$\Delta$ Week 12 , baseline & 107 & $-8(-4$ to -11$)$ & 110 & $-7(-4$ to -11$)$ & $0(-5$ to 5$)$ \\
\hline$\Delta$ Week 24, baseline & 108 & $-8(-5$ to -12$)$ & 110 & $-8(-3$ to -12$)$ & $0(-6$ to 5$)$ \\
\hline \multicolumn{6}{|l|}{ WOMAC stiffness } \\
\hline Baseline (SD) & 115 & $58(22)$ & 113 & $54(19)$ & \\
\hline$\Delta$ Week 12 , baseline & 106 & $-7(-3$ to -11$)$ & 110 & $-9(-5$ to -13$)$ & $2(-4$ to 7$)$ \\
\hline$\Delta$ Week 24 , baseline & 108 & $-5(-1$ to -8$)$ & 107 & $-7(-3$ to -12$)$ & $3(-3$ to 8$)$ \\
\hline \multicolumn{6}{|l|}{ WOMAC function } \\
\hline Baseline (SD) & 112 & $52(18)$ & 115 & $47(17)$ & \\
\hline$\Delta$ Week 12 , baseline & 104 & $-7(-4$ to -10$)$ & 109 & $-7(-3$ to -10$)$ & $-1(-5$ to 4$)$ \\
\hline$\Delta$ Week 24 , baseline & 103 & $-8(-4$ to -11$)$ & 107 & $-7(-3$ to -10$)$ & $-1(-6$ to 4$)$ \\
\hline \multicolumn{6}{|l|}{ VAS-PGA } \\
\hline Baseline (SD) & 112 & $52(25)$ & 115 & $53(26)$ & \\
\hline$\Delta$ Week 12 , baseline & 100 & $-7(-1$ to -13$)$ & 106 & $-9(-3$ to -15$)$ & $1(-7$ to 10$)$ \\
\hline$\Delta$ Week 24 , baseline & 95 & $-7(-1$ to -13$)$ & 105 & $-10(-3$ to -17$)$ & $3(-6$ to 12$)$ \\
\hline \multicolumn{6}{|c|}{ PCS (possible range 4-71)* } \\
\hline Baseline (SD) & 114 & $36(8)$ & 114 & $36(7)$ & \\
\hline$\Delta$ Week 12 , baseline ${ }^{\dagger}$ & 104 & $2(0$ to 3$)$ & 109 & $3(1$ to 4$)$ & $-1(-3$ to 1$)$ \\
\hline$\Delta$ Week 24 , baseline $^{\dagger}$ & 105 & $2(1$ to 3$)$ & 108 & $2(1$ to 4$)$ & $0(-2$ to 3$)$ \\
\hline \multicolumn{6}{|c|}{ MCS (possible range $2-74)^{*}$} \\
\hline Baseline (SD) & 114 & $52(11)$ & 114 & $54(11)$ & \\
\hline$\Delta$ Week 12 , baseline $^{\dagger}$ & 104 & $1(-1$ to 2$)$ & 109 & $-1(-3$ to 1$)$ & $2(0$ to 4$)$ \\
\hline$\Delta$ Week 24 , baseline $^{\dagger}$ & 105 & $1(-1$ to 2$)$ & 108 & $0(-1$ to 2$)$ & $0(-2$ to 3$)$ \\
\hline
\end{tabular}

Negative signs indicate improvement within groups (unless stated otherwise) or improvement in favour of doxycycline (in case of differences in change between groups). Scores are normalised (0-100; $0=$ no symptoms) unless stated otherwise.

${ }^{*}$ Norm-based scores, higher scores indicate better health, individual scores in the $45-55$ range indicate average health.

${ }^{\dagger}$ Negative signs indicate deterioration within groups or improvement in favour of placebo (in case of differences in change between groups).

MCS, mental component score; PCS, physical component score, VAS-PGA, visual analogue scale-patient global assessment; WOMAC Western Ontario McMaster Universities. 
taking doxycycline ceased treatment because of side effects (19 vs five, $\mathrm{p}<0.01)$. Adverse events in both treatment groups reported by more than two subjects are shown in table 3 .

The only adverse event that occurred significantly more often in one of the treatment groups was sun sensitivity. The cumulative incidence of upper respiratory tract infection was, however, somewhat lower in doxycycline-treated patients, although this did not reach significance. The development of laboratory abnormalities was rare and did not significantly differ between the treatment groups. In seven subjects (five doxycycline vs two placebo group) low-normal vitamin B12 levels were measured at the end of the study $(p=0.3)$. A total of five SAE occurred during the trial: one traumatic patella fracture (placebo group); two myocardial infarctions (both doxycycline group); one total knee replacement (doxycycline group) and one arthroscopic meniscectomy (placebo group). None of the SAE were likely to have been attributable to doxycycline, therefore no suspected unexpected serious adverse reactions occurred during the trial.

\section{Sensitivity analyses}

Analysis regarding the proportion of responders in subjects who completed the trial per protocol yielded the same results as the primary analysis. Also, an analysis of (non-)response in adherent participants resulted in similar findings.

\section{DISCUSSION}

The findings of the present study indicate that doxycycline is not effective in reducing symptoms in knee osteoarthritis patients over a 24 -week study period, but is associated with an increased risk of adverse events. Although previously a possible structure-modifying effect of doxycycline was suggested, this is not accompanied by symptom relief in the short and medium term. Because of the unfavourable risk-benefit ratio, doxycycline should not be used in the management of knee osteoarthritis.

The lack of effect seen in this study is not caused by insufficient adherence to the medication. Although pill counts showed that approximately $20 \%$ of the patients did not reach the target of taking $80 \%$ or more of the study medication, subanalysis in adherent participants yielded the same findings. Although varus malalignment may have negated the symptom-modifying effect of doxycycline, as reported for the structure-modifying effects of doxycycline, ${ }^{29}$ this seems very unlikely as major malalignment was one of the exclusion criteria.

Generalisability seems to be fair as the study group is comparable with other cohorts of knee osteoarthritis consisting mainly of middle-aged obese women. ${ }^{30}{ }^{31}$ In this study a relatively low frequency of subjects experienced severe knee pain (16\%). This is in contrast to other more symptomatic knee osteoarthritis cohort studies, like for example the secondary care CONTROLPRO cohort, in which $54 \%$ of knee osteoarthritis patients experienced severe pain. ${ }^{32}$ However, stratified analyses in patients with severe pain did not show any trend for a symptom-reducing effect of doxycycline. Also, results of our study possibly cannot be extrapolated to knee osteoarthritis patients with low or high radiographic $\mathrm{K} \& \mathrm{~L}$ scores (ie, $\leq 1$ or $>3$ ). It could be possible that we included participants with slightly less extensive radiographic osteoarthritis compared with other studies, because radiographs obtained in the flexion view may have a higher sensitivity for osteoarthritis-related radiographic changes compared with conventional AP views. ${ }^{33}$ However, this does not challenge the validity of the present study as it does not compromise the external validity, because in both K\&L groups a lack of response was observed indicating absence of effect modification.

To our knowledge, up to now no other studies have evaluated the effects of doxycycline on symptoms caused by osteoarthritis. As mentioned earlier, the only study of doxycycline in human knee osteoarthritis ${ }^{13}$ indicated that this agent retards radiographic progression of knee osteoarthritis, although the primary goal of the study-reduction of progression of less extensive knee osteoarthritis, was not met. A trend to pain reduction was also seen, possibly reflecting the anti-inflammatory effect of tetracyclines as studied for minocycline in rheumatoid arthritis. ${ }^{34}$

Although doxycycline (as a metalloproteinase inhibitor) does not seem to be a symptom modifier in the short and medium term in knee osteoarthritis patients with moderate pain, this agent could still have potential structure-modifying effects. However, the use of doxycycline for a longer period has some drawbacks regarding side effects (mainly sun sensitivity) and possibly the development of antimicrobacterial resistance. Therefore, efforts have been made to develop synthetic metalloproteinase inhibitors, ${ }^{35} 36$ although until now without a favourable risk-benefit ratio.

Future research should give insight into the structuremodifying properties of the inhibition of metalloproteinases

Table 3 Adverse events in both study groups

\begin{tabular}{|c|c|c|c|c|c|c|c|c|}
\hline \multirow[b]{2}{*}{ Adverse event } & \multirow{2}{*}{$\begin{array}{l}\text { Total study } \\
\text { population } \\
(\mathrm{n}=232), \mathrm{n}(\%)^{*}\end{array}$} & \multirow[b]{2}{*}{$\begin{array}{l}\text { Doxycycline } \\
(n=116), n(\%)\end{array}$} & \multirow[b]{2}{*}{$\begin{array}{l}\text { Placebo } \\
(n=116), n(\%)\end{array}$} & \multirow[b]{2}{*}{ p Value } & \multicolumn{2}{|l|}{ Doxycycline } & \multicolumn{2}{|l|}{ Placebo } \\
\hline & & & & & $\begin{array}{l}\text { Led to temporarily } \\
\text { discontinuation, } \mathrm{n}^{\dagger}\end{array}$ & $\begin{array}{l}\text { Led to permanent } \\
\text { discontinuation, } n\end{array}$ & $\begin{array}{l}\text { Led to temporarily } \\
\text { discontinuation, } n\end{array}$ & $\begin{array}{l}\text { Led to permanent } \\
\text { discontinuation, } n\end{array}$ \\
\hline Sun sensitivity & $38(16)$ & $35(30)$ & $3(3)$ & $<0.001$ & 6 & 9 & 0 & 1 \\
\hline Diarrhoea & $25(11)$ & $15(13)$ & $10(9)$ & 0.40 & 1 & 5 & 0 & 1 \\
\hline $\begin{array}{l}\text { Abdominal } \\
\text { discomfort }\end{array}$ & $16(7)$ & $6(5)$ & $10(9)$ & 0.44 & 0 & 4 & 0 & 1 \\
\hline Erythema & $12(5)$ & $8(7)$ & $4(3)$ & 0.38 & 1 & 2 & 0 & 1 \\
\hline Nausea & $10(4)$ & $5(4)$ & $5(4)$ & 1.0 & 0 & 1 & 0 & 1 \\
\hline $\begin{array}{l}\text { Upper respiratory } \\
\text { tract infection }\end{array}$ & $9(4)$ & $2(2)$ & $7(6)$ & 0.17 & 1 & 0 & 1 & 0 \\
\hline Arthralgia/myalgia & $8(3)$ & $4(3)$ & $4(3)$ & 1.0 & 0 & 2 & 0 & 0 \\
\hline Headache & $7(3)$ & $4(3)$ & $3(3)$ & 1.0 & 0 & 0 & 1 & 0 \\
\hline Oedema & $6(3)$ & $3(3)$ & $3(3)$ & 1.0 & 0 & 0 & 0 & 0 \\
\hline Constipation & $6(3)$ & $4(3)$ & $2(2)$ & 0.68 & 1 & 1 & 0 & 0 \\
\hline Mycosis & $5(2)$ & $4(3)$ & $1(1)$ & 0.37 & 1 & 1 & 0 & 0 \\
\hline Other & $26(11)$ & $10(9)$ & $16(14)$ & 0.30 & 2 & 2 & 1 & 2 \\
\hline
\end{tabular}

* Subjects could have reported more than one adverse event.

${ }^{\dagger}$ Subjects could have ceased treatment because of more than one adverse event. 
in human osteoarthritis. Regarding symptom modifying in osteoarthritis, improvement of the strategies of existing treatment modalities ${ }^{37}$ or the development of new classes of analgesics $^{38}$ will probably result in better outcomes in patients with symptomatic osteoarthritis.

In conclusion, the present study showed that twice daily doxycycline for 24 weeks did not have any effect on symptoms in knee osteoarthritis patients, compared with placebo. Therefore, doxycycline should not be applied as a symptom modifier in knee osteoarthritis.

Acknowledgements The authors thank Dr BJF van den Bemt for study medication supply, FW Snijders and NWD Eikelenboom for data entry and management, and VHHP Straten and MA Arts-Sanders for patient screening.

Competing interests None.

Patient consent Obtained.

Ethics approval This study was conducted with the approval of the local Medical Research Ethics Committee, region Arnhem/Nijmegen, The Netherlands, and the national Central Committee on Research involving Human Subjects (CCMO).

Provenance and peer review Not commissioned; externally peer reviewed.

\section{REFERENCES}

1. Sengupta K, Alluri KV, Satish AR, et al. A double blind, randomized, placebo controlled study of the efficacy and safety of 5-Loxin for treatment of osteoarthritis of the knee. Arthritis Res Ther 2008;10:R85.

2. Clegg DO, Reda DJ, Harris CL, et al. Glucosamine, chondroitin sulfate, and the two in combination for painful knee osteoarthritis. N Engl J Med 2006;354:795-808.

3. Rozendaal RM, Koes BW, van Osch GJ, et al. Effect of glucosamine sulfate on hip osteoarthritis: a randomized trial. Ann Intern Med 2008;148:268-77.

4. Kahan A, Uebelhart D, De Vathaire F, et al. Long-term effects of chondroitins 4 and 6 sulfate on knee osteoarthritis: the study on osteoarthritis progression prevention, a two-year, randomized, double-blind, placebo-controlled trial. Arthritis Rheum 2009:60:524-33

5. Dougados M, Nguyen M, Berdah L, et al. Evaluation of the structure-modifying effects of diacerein in hip osteoarthritis: ECHODIAH, a three-year, placebo-controlled trial. Evaluation of the Chondromodulating Effect of Diacerein in $\mathrm{OA}$ of the Hip. Arthritis Rheum 2001;44:2539-47.

6. Bruyere 0, Delferriere D, Roux C, et al. Effects of strontium ranelate on spinal osteoarthritis progression. Ann Rheum Dis 2008;67:335-9.

7. Güler-Yüksel M, Allaart CF, Watt I, et al. Treatment with TNF-a inhibitor infliximab might reduce hand osteoarthritis in patients with rheumatoid arthritis. Osteoarthr Cartil 2010;18:1256-62.

8. Fioravanti A, Fabbroni M, Cerase A, et al. Treatment of erosive osteoarthritis of the hands by intra-articular infliximab injections: a pilot study. Rheumatol Int 2009;29:961-5.

9. Chevalier $\mathbf{X}$, Goupille $P$, Beaulieu $A D$, et al. Intraarticular injection of anakinra in osteoarthritis of the knee: a multicenter, randomized, double-blind, placebocontrolled study. Arthritis Rheum 2009;61:344-52.

10. Baltzer AW, Moser C, Jansen SA, et al. Autologous conditioned serum (Orthokine) is an effective treatment for knee osteoarthritis. Osteoarthr Cartil 2009;17:152-60.

11. Manicourt DH, Azria M, Mindeholm L, et al. Oral salmon calcitonin reduces Lequesne's algofunctional index scores and decreases urinary and serum levels of biomarkers of joint metabolism in knee osteoarthritis. Arthritis Rheum 2006;54:3205-11.

12. Spector TD, Conaghan PG, Buckland-Wright JC, et al. Effect of risedronate on joint structure and symptoms of knee osteoarthritis: results of the BRISK randomized, controlled trial [ISRCTN01928173]. Arthritis Res Ther 2005;7:R625-33.

13. Brandt KD, Mazzuca SA, Katz BP, et al. Effects of doxycycline on progression of osteoarthritis: results of a randomized, placebo-controlled, double-blind trial. Arthritis Rheum 2005;52:2015-25.

14. Yang KG, Raijmakers NJ, van Arkel ER, et al. Autologous interleukin-1 receptor antagonist improves function and symptoms in osteoarthritis when compared to placebo in a prospective randomized controlled trial. Osteoarthr Cartil 2008;16:498-505.

15. Pavelka K, Trc T, Karpas K, et al. The efficacy and safety of diacerein in the treatment of painful osteoarthritis of the knee: a randomized, multicenter, doubleblind, placebo-controlled study with primary end points at two months after the end of a three-month treatment period. Arthritis Rheum 2007:56:4055-64.
16. Lequesne M, Maheu E, Cadet $\mathbf{C}$, et al. Structural effect of avocado/soybean unsaponifiables on joint space loss in osteoarthritis of the hip. Arthritis Rheum 2002;47:50-8.

17. Maheu E, Mazières B, Valat JP, et al. Symptomatic efficacy of avocado/soybean unsaponifiables in the treatment of osteoarthritis of the knee and hip: a prospective, randomized, double-blind, placebo-controlled, multicenter clinical trial with a six-month treatment period and a two-month followup demonstrating a persistent effect. Arthritis Rheum 1998;41:81-91.

18. Rossini M, Viapiana O, Ramonda R, et al. Intra-articular clodronate for the treatment of knee osteoarthritis: dose ranging study vs hyaluronic acid. Rheumatology (Oxford) 2009;48:773-8.

19. Bingham CO, III, Buckland-Wright JC, Garnero P, et al. Risedronate decreases biochemical markers of cartilage degradation but does not decrease symptoms or slow radiographic progression in patients with medial compartment osteoarthritis of the knee: results of the two-year multinational knee osteoarthritis structural arthritis study. Arthritis Rheum 2006;54:3494-507.

20. Yu LP, Jr, Smith GN, Jr, Brandt KD, et al. Reduction of the severity of canine osteoarthritis by prophylactic treatment with oral doxycycline. Arthritis Rheum 1992:35:1150-9

21. Smith GN, Jr, Yu LP, Jr, Brandt KD, et al. Oral administration of doxycycline reduces collagenase and gelatinase activities in extracts of human osteoarthritic cartilage. J Rheumatol 1998;25:532-5.

22. Smith GN, Jr, Mickler EA, Hasty KA, et al. Specificity of inhibition of matrix metalloproteinase activity by doxycycline: relationship to structure of the enzyme. Arthritis Rheum 1999:42:1140-6.

23. Altman R, Asch E, Bloch D, et al. Development of criteria for the classification and reporting of osteoarthritis. Classification of osteoarthritis of the knee. Diagnostic and Therapeutic Criteria Committee of the American Rheumatism Association. Arthritis Rheum 1986;29:1039-49.

24. Kellgren JH, Lawrence JS. Radiological assessment of osteo-arthrosis. Ann Rheum Dis 1957;16:494-502.

25. de Groot IB, Favejee MM, Reijman M, et al. The Dutch version of the Knee Injury and Osteoarthritis Outcome Score: a validation study. Health Qual Life Outcomes 2008;6:16

26. Bellamy N, Buchanan WW, Goldsmith $\mathrm{CH}$, et al. Validation study of WOMAC: a health status instrument for measuring clinically important patient relevant outcomes to antirheumatic drug therapy in patients with osteoarthritis of the hip or knee. $J$ Rheumatol 1988:15:1833-40.

27. Ware JE, Jr, Sherbourne CD. The MOS 36-item short-form health survey (SF-36). I. Conceptual framework and item selection. Med Care 1992;30:473-83.

28. Pham T, van der Heijde D, Altman RD, et al. OMERACT-OARSI initiative: Osteoarthritis Research Society International set of responder criteria for osteoarthritis clinical trials revisited. Osteoarthr Cartil 2004;12:389-99.

29. Mazzuca SA, Brandt KD, Chakr R, et al. Varus malalignment negates the structuremodifying benefits of doxycycline in obese women with knee osteoarthritis. Osteoarthr Cartil 2010;18:1008-11.

30. Wesseling J, Dekker J, van den Berg WB, et al. CHECK (Cohort Hip and Cohort Knee): similarities and differences with the Osteoarthritis Initiative. Ann Rheum Dis 2009:68:1413-19.

31. van Dijk GM, Veenhof C, Spreeuwenberg P, et al. Prognosis of limitations in activities in osteoarthritis of the hip or knee: a 3-year cohort study. Arch Phys Med Rehabil 2010:91:58-66.

32. Snijders GF, den Broeder AA, van Riel PL, et al. Evidence-based conservative treatment of knee and hip osteoarthritis: between knowing and doing. Scand J Rheumatol. Published Online First: 25 January 2011 doi:10.3109/03009742.2010.530611.

33. Mazzuca SA, Brandt KD, German NC, et al. Development of radiographic changes of osteoarthritis in the "Chingford knee" reflects progression of disease or nonstandardised positioning of the joint rather than incident disease. Ann Rheum Dis 2003:62:1061-5.

34. Kloppenburg M, Breedveld FC, Terwiel JP, et al. Minocycline in active rheumatoid arthritis. A double-blind, placebo-controlled trial. Arthritis Rheum 1994;37:629-36.

35. Krzeski P, Buckland-Wright C, Bálint G, et al. Development of musculoskeletal toxicity without clear benefit after administration of PG-116800, a matrix metalloproteinase inhibitor, to patients with knee osteoarthritis: a randomized, 12-month, double-blind, placebo-controlled study. Arthritis Res Ther 2007;9:R109.

36. Baragi VM, Becher G, Bendele AM, et al. A new class of potent matrix metalloproteinase 13 inhibitors for potential treatment of osteoarthritis: evidence of histologic and clinical efficacy without musculoskeletal toxicity in rat models. Arthritis Rheum 2009:60:2008-18.

37. van den Ende CM, Bierma-Zeinstra SM, Vlieland TP, et al. [Conservative treatment of hip and knee osteoarthritis: a systematic, step-by-step treatment strategy]. Ned Tijdschr Geneeskd 2010;154:A1574.

38. Lane NE, Schnitzer TJ, Birbara CA, et al. Tanezumab for the treatment of pain from osteoarthritis of the knee. N Engl J Med 2010;363:1521-31. 